\title{
Abraham Lincoln Association
}

\section{BOARD OF DIRECTORS}

KenNeth L. Anderson

J. Steven Beckett

Roger D. Billings, JR.

Roger D. BRIDGES

Julie Cellini

Joshua A. Claybourn

ROBERT J. DAVIS

Chris DeRose

JIM EDGAR

Guy C. Fraker

Sara Vaughn Gabbard

JOSEPH GARRERA

Donald R. GRAHAM

Allen C. Guelzo

RICHARD E. HART

DAVID JoENS

Ron J. KeLler

SUSAN J. Koch

Michelle A. KROWL

ROBERT J. LENZ
Sue S. Massie

Dan Monroe

AnNe Moseley

KeRI L. NeKrasz

James W. Patton III

Tiffany A. Player

Mark POHLAD

ROGER RUdich

SCOTT T. SCHROEDER

WILLIAM G. SHEPHERD

RoNALD D. SPEARS

BRIAN STEENBERgEN

Robert A. Stuart, Jr.

JAMES L. SWANSON

LOUISE TAPER

Donald R. TRACY

DANIEL R. WEINBERG

JONATHAN W. WHITE

BARBARA WYSOCKI

STEPHEN M. Young

\section{HONORARY DIRECTORS}

President Joseph R. Biden JR.

Governor J. B. PritzKer

Lt. Governor Juliana Stratton

SENATOR Richard Durbin

Senator Tammy Duckworth

Representative Darin LaHood Representative Mary Miller

RePresentative Rodney Davis

Chief Justice Anne M. Burke

MAYOR JAMES LANGFELDER

EMERITUS DIRECTORS

Cullom Davis Richard Mills

Mark A. Plummer N. Ronald Thunman

DISTINGUISHED DIRECTORS

Doris Kearns Goodwin

THOMAS F. SCHWARTZ
LEWIS E. LEHRMAN

WAYNe C. TEMPLE

To learn more about the Abraham Lincoln Association or to become a member, please visit our website at www.abrahamlincolnassociation.org. 